\title{
A NOVEL DWELLING TIME DESIGN METHOD FOR LOW PROBABILITY OF INTERCEPT IN A COMPLEX RADAR NETWORK
}

\author{
Z. ZHANG ${ }^{1,2,3}$, J. ZHU ${ }^{1}$ \& S. SALOUS ${ }^{3}$ \\ ${ }^{1}$ Science and Technology on Electronic Information, Control Laboratory, People's Republic of China. \\ ${ }^{2}$ College of Electronic Information, JiangSu University of Science and Technology, People's Republic of China. \\ ${ }^{3}$ School of Engineering and Computing Sciences, Durham University, UK.
}

\section{ABSTRACT}

To achieve the important tactical requirement of low probability of intercept (LPI) in the complex radar network, dynamically controlling the emission of the radars is very necessary. A novel radar dwelling time control strategy based on an interacting multiple model algorithm is presented in this paper, which controls the dwelling time of radar according to predicted covariance matrix during tracking, taking advantage of the relation model between the dwelling time and the tracking performance. First, the complex radar network is built for target tracking. Secondly, the influence of the dwelling time is considered in the tracking performance of the complex radar network. Finally, a decision will be made after the dwelling time for every radar is obtained by particle swarm optimization, the radar with the smallest dwelling time will be selected to track target. The tracking accuracy and LPI performance are demonstrated in the Monte Carlo simulations. The results are validated through the comparison with other methods.

Keywords: dwelling time, interacting multiple model, low probability of intercept, target tracking.

\section{INTRODUCTION}

As we know, the less the emitted time of the radar, the more the excellent performance of the low probability of intercept (LPI) [1]. To achieve the important requirement of LPI, dynamically scheduling and controlling the dwelling time of the radar during the sensor management is very necessary. The work in [2] formalizes the typical workload of a modern-phased array radar and proposes a rate-based approach to schedule radar dwells in a real-time manner. The paper [3] develops a generalized framework for the radar task scheduling problem as an optimization model, and all radar task parameters are treated as variables, thereby allowing greater scheduling flexibility and the ability to handle more targets using a single radar. The scheduling of track dwells to minimize radar energy and time with an agile beam radar is considered in [4], where the trade between higher energy waveforms and radar time is further investigated. The algorithm in [5] introduces time windows that specify allowable earliness and lateness of radar tasks, and proposes a chaining process that combines the dwell times and the time windows of tasks with consecutive priorities. The work of Severson and Paley [6] presents a distributed, consensus-based approach to optimize radar resource management for ballistic missile surveillance and tracking.

Almost all of those works concern the performance of the single radar, and there are few studies on the design of dwelling time and radars selection in the complex radar network for excellent LPI performance. In this paper, a novel scheduling algorithm for dwelling time in radar networks is proposed. The remainder of this paper is organized as follows. Sections 2 and 3 describe the interacting multiple model (IMM) algorithm of target tracking and particle swarm optimization (PSO), respectively. Section 4 presents the dwelling time scheduling method in sensor network in detail. Simulations of the proposed algorithms and comparison results with other methods are provided in Section 5. The conclusions are presented in Section 6. 


\section{TARGET TRACKING ALGORITHM BASED ON IMM}

IMM method is used for tracking maneuvering target in this paper. Many dynamic models are used for matching different motion states, and the switch probability of a different model is a Markov chain. Kalman filtering is employed for the estimation of target state and the update of model probability in the algorithm, and all dynamic models are parallel processed and the model probability represents model switch [7].

All the dynamic models are: $M=\left\{m^{1}, m^{2}, \ldots, m^{r}\right\}, m_{k}^{i}$ is the $i$ th model used at time $k$, the switch probability from model $m_{k}^{j}$ to model $m_{k+1}^{i}$ is $P\left\{m_{k+1}^{j} \mid m_{k}^{i}\right\}=\pi_{i j}, m_{k+1}^{j}, m_{k}^{i} \in M, \sum_{i=1}^{r} \pi_{i j}=1, i=1,2, \ldots, r$ $\mu_{j}(k)$ is the probability of model $j, \mu_{j}(k)=P\left\{m(k)=j \mid Z^{k}\right\}$.

Let $X(k)$ and $Z(k)$ represent the state vector and the observation vector, respectively; the state equation and transfer equation at time $k$ are:

$$
\boldsymbol{X}(k+1)=\phi_{j}(k+1) \boldsymbol{X}(k)+\boldsymbol{w}_{j}(k)
$$

and

$$
\boldsymbol{Z}(k)=\boldsymbol{H}_{j} \boldsymbol{X}(k)+\boldsymbol{v}_{j}(k)
$$

where $w_{j}(k)$ and $v_{j}(k)$ are stationary white-noise processes with covariance matrices $Q_{j}(k)$ and $W(k)$ of model $j, \phi_{j}(k)$ is the transition matrix and $H_{j}$ is the observation matrix.

Every recurrence of the IMM algorithm contains: interacting of input, model's filtering, update of model probability and interacting of output.

\subsection{Interacting of input}

Utilizing all the states and model probabilities from last recurrence, the computation of input state $\hat{\mathrm{X}}_{0 j}\left(t_{k-1}\right)$ and covariance $\mathrm{P}_{0 j}\left(t_{k-1}\right)$ of model $j$ can be expressed as

$$
\begin{gathered}
\hat{\boldsymbol{X}}_{0 j}(k-1)=\sum_{i=1}^{r} \hat{\boldsymbol{X}}_{i}(k-1) \boldsymbol{\mu}_{i \mid j}(k-1) \\
\boldsymbol{P}_{0 j}(k-1)=\sum_{i=1}^{r}\left(\boldsymbol{P}_{i}(k-1)+\boldsymbol{\alpha} \cdot \boldsymbol{\alpha}^{T}\right) \cdot \boldsymbol{\mu}_{i \mid j}(k-1) \\
\boldsymbol{\alpha}=\left[\hat{\boldsymbol{X}}_{i}(k-1)-\hat{\boldsymbol{X}}_{0 j}(k-1)\right] \\
\boldsymbol{\mu}_{i \mid j}(k-1)=\boldsymbol{P}\left\{\boldsymbol{M}(k-1)=i \mid \boldsymbol{M}(k)=j, \boldsymbol{Z}^{k-1}\right\}=\frac{\pi_{i j} \boldsymbol{\mu}_{i}(k-1)}{\sum_{i=1}^{r} \pi_{i j} \boldsymbol{\mu}_{i}(k-1)}
\end{gathered}
$$

2.2 Model's filtering

In the light of step (1) and observation data $Z(k)$, the Kalman filtering of every model is as follows:

The prediction of state and covariance for model $j$ has the form of

$$
\begin{gathered}
\hat{\boldsymbol{X}}_{j}^{\prime}(k)=\phi_{j}(k) \hat{\boldsymbol{X}}_{0 j}(k-1) \\
\boldsymbol{P}_{j}^{\prime}(k)=\phi_{j}(k) \boldsymbol{P}_{0 j}(k-1)\left(\phi_{j}(k)\right)^{T}+\boldsymbol{Q}_{j}
\end{gathered}
$$

The residual error represented by observation data and estimation of last observation is 


$$
\boldsymbol{v}_{j}(k)=\boldsymbol{Z}(k)-\boldsymbol{H}_{j} \hat{\boldsymbol{X}}_{j}^{\prime}(k)
$$

The variance matrix of the residual error is obtained by the follow equation:

$$
\boldsymbol{S}_{j}(k)=\boldsymbol{H}_{j} \boldsymbol{P}_{j}^{\prime}(k)\left(\boldsymbol{H}_{j}\right)^{T}+\boldsymbol{W}_{j}(k)
$$

Computation of the filtering gain is given as

$$
\boldsymbol{K}_{j}(k)=\boldsymbol{P}_{j}^{\prime}(k)\left(\boldsymbol{H}_{j}\right)^{T}\left(\boldsymbol{S}_{j}(k)\right)^{-1}
$$

Equation of updated state is

$$
\boldsymbol{P}_{j}(k)=\left(\boldsymbol{I}-\boldsymbol{K}_{j}(k) \boldsymbol{H}_{j}\right) \boldsymbol{P}_{j}^{\prime}(k)
$$

Estimation of the covariance is

$$
P_{j}(k)=\left(I-K_{j}(k) H_{j}\right) P_{j}^{\prime}(k)
$$

2.3 Updating of the model probability

The model probability is recursively updated as

$$
\boldsymbol{\mu}_{j}(k)=\frac{\boldsymbol{\Lambda}_{j}(k) \sum_{i=1}^{r} \pi_{i j} \boldsymbol{\mu}_{i}(k-1)}{\sum_{j=1}^{r} \sum_{i=1}^{r} \boldsymbol{\Lambda}_{j}(k) \pi_{i j} \boldsymbol{\mu}_{i}(k-1)}
$$

$\Lambda_{j}(k)$ is the likelihood function of model $j$ at time $k$.

\subsection{Interacting of output}

The final estimation of state vector from all the models' state is

$$
\hat{\boldsymbol{X}}(k)=\sum_{j=1}^{r} \hat{\boldsymbol{X}}_{j}(k) \boldsymbol{\mu}_{j}(k)
$$

Estimated matrix of covariance is derived as

$$
\boldsymbol{P}(k)=\sum_{j=1}^{r} \boldsymbol{\mu}_{j}(k)\left(\boldsymbol{P}_{j}(k)+\boldsymbol{b} \boldsymbol{b}^{T}\right)
$$

where $\boldsymbol{b}=\left[\hat{\boldsymbol{X}}_{j}(k)-\hat{\boldsymbol{X}}(k)\right]$.

\section{PARTICLE SWARM OPTIMIZATION (PSO)}

PSO algorithm proposed by Kennedy and Eberhart [8] has been widely used in many fields. It is an evolutionary algorithm according to social interaction between independent particles. In PSO method, every potential solution for optimization problem is supposed as a point called particle in the space. The swarm composed by $N$ particles moves through the problem space with the moving velocity of each particle. The position and velocity of the $i$ th particle is represented as $\boldsymbol{x}_{i}=\left(x_{i 1}, x_{i 2}, \ldots\right.$, $\left.x_{i N}\right)$ and $v_{i}=\left(v_{i 1}, v_{i 2, \ldots}, v_{i N}\right)$, respectively, where $N$ is the dimension of the space.

The $i$ th particle tracks its personal best position (pbest) as a vector $\boldsymbol{p}_{i}=\left(p_{i 1}, p_{i 2, \ldots,}, p_{i N}\right)$; the global best position (gbest) among all the particles is represented as $\boldsymbol{p}_{g}=\left(p_{g 1,} p_{g 2, \ldots}, p_{g N}\right)$. 
Usually the optimization problem can be formulated as follows:

$$
\min f(X) \text {, subject to } g(x) \geq 0
$$

where $\boldsymbol{X}=\left(x_{1}, x_{2}, \ldots, x_{m}\right)$.

The steps of PSO method are outlined as follows:

1. Generating the original particle position and velocity.

2. Exceeding boundary control.

3. Computing the fitness function $f(X)$ for each particle.

4. Updating the pbest:

$$
\text { pbest }_{i}^{k+1}=\left\{\begin{array}{l}
x_{i}^{k+1} \quad \text { if } \boldsymbol{f}\left(x_{i}^{k+1}\right)<\boldsymbol{f}\left(\text { pbest }_{i}^{k}\right) \\
\text { pbest } t_{i}^{k} \text { otherwise }
\end{array}\right.
$$

5. Updating the gbest $_{i}$ :

$$
\text { gbest }_{i}^{k+1}=\left\{\begin{array}{l}
\text { pbest }_{i}^{k+1} \text { if } \boldsymbol{f}\left(x_{i}^{k+1}\right)<\boldsymbol{f}\left(\text { pbest }_{i}^{k}\right) \\
\text { gbest }_{i}^{k} \text { otherwise }
\end{array}\right.
$$

6. Updating the position and velocity:

$$
\begin{gathered}
v_{i d}^{k+1}=w \cdot v_{i d}^{k}+c_{1} \cdot r_{1} \cdot\left(\text { pbest } t_{i d}^{k}-x_{i d}^{k}\right) \\
+c_{2} \cdot r_{2} \cdot\left(\text { gbest } t_{i d}^{k}-x_{i d}^{k}\right) \\
x_{i d}^{k+1}=x_{i d}^{k}+v_{i d}^{k+1}
\end{gathered}
$$

where $i=1,2, \ldots n, d=1,2 \ldots m, n$ and $m$ are the number of particles and dimensions in the space, $w$ represents the inertia weight factor which is between 0.4 and $0.9, c_{1}$ and $c_{2}$ are acceleration constants usually selected as $2, r_{1}$ and $r_{2}$ are uniformly distributed random numbers in $(0,1)$.

7. Return to step (2) and go on the iteration.

\section{DWELLING TIME ALLOCATION ALGORITHM BASED ON PSO}

4.1 Design for covariance matrix $W_{k}$ of measurement noise

The covariance matrix $W_{k}$ of measurement noise is controlled by the emitted energy. As we know, radar equation at time $k$ is as follows:

$$
R_{k}^{4}=t_{B}^{k} \frac{P_{a v}^{k} G_{T} G_{R} \lambda^{2} \sigma_{k}}{(4 \pi)^{3} K T_{R} S_{N R}^{k} L}
$$

where $t_{B}^{k}$ is the single dwelling time of the beam from the normal direction at time $k, P_{\alpha v}^{k}$ is the average radiated power, $G_{R}$ is the receiver gain, $\mathrm{s}_{k}$ is the radar cross section of the target, $K$ is the Boltzmann constant, $T_{R}$ and $L$ are, respectively, effective noise temperature and radar system loss, $R_{k}$ is the detection range, $G_{T}$ is the transmit gain, $S_{N R}^{k}$ represents the signal-to-noise ratio of the system at time $k$. Suppose when the target whose range is $R_{0}$, the radar has to emit the power $P_{a v 0}$, and the radar equation becomes 


$$
R_{0}^{4}=t_{B 0} \frac{P_{a v 0} G_{T} G_{R} \lambda^{2} \sigma_{0}}{(4 \pi)^{3} K T_{R} S_{N R 0} L}
$$

Combining (22) with (23), the emitted signal-to-noise ratio at time $k$ can be written as

$$
S_{N R}^{k}=\frac{t_{B}^{k} P_{a v}^{k} S_{N R 0}}{t_{B 0} P_{a v 0}} \frac{R_{0}^{4}}{R_{k}^{4}}
$$

where the emitted power is supposed to be a constant parameter in this paper.

The single pulse signal is radiated by the radar, and the covariance of the measurement noise can be denoted as

$$
\boldsymbol{W}=\left[\begin{array}{ll}
\frac{c^{2} T_{p}}{8 S_{N R}^{k}} & 0 \\
0 & \frac{3 c^{2}}{w_{c}^{2} T_{p}^{2} S_{N R}^{k}}
\end{array}\right]
$$

where $T_{p}$ is the pulse width, $c$ is the wave velocity, and $w_{c}$ is the carrier frequency. We can see that different $t_{B}^{k}$ can lead to different $\boldsymbol{W}$. However, during the tracking process, $R_{k}$ is unknown before radar detection. Therefore, $R_{k}$ in (24) is replaced by $R_{k}^{\text {pre }}$, which is predicted by $R_{k-1}$ and $v_{k-1} \cdot R_{k}^{\text {pre }}$ is presented as

$$
R_{k}^{\text {pre }}=R_{k-1}+v_{k-1} T
$$

$R_{k-1}$ and $v_{k-1}$ are the target's range and velocity, which are estimated by the IMM tracking algorithm at time $k-1$, and $T$ is the tracking interval.

\subsection{Computation of predicted tracking covariance matrix}

The prediction of covariance for model $j$ at time $k$ can be represented as

$$
\boldsymbol{P}_{j}^{\prime}(k+1)=\phi_{j}(k+1) \boldsymbol{P}_{0 j}(k)\left(\phi_{j}(k+1)\right)^{T}+\boldsymbol{Q}_{j}
$$

Then, the variance matrix $S_{j}(k+1)$ and filtering gain $K_{j}(k+1)$ can be written, respectively,

$$
\begin{gathered}
\boldsymbol{S}_{j}(k+1)=\boldsymbol{H}_{j} \boldsymbol{P}_{j}^{\prime}(k+1)\left(\boldsymbol{H}_{j}\right)^{T}+\boldsymbol{W}_{j}(k+1) \\
\boldsymbol{K}_{j}(k+1)=\boldsymbol{P}_{j}^{\prime}(k+1)\left(\boldsymbol{H}_{j}\right)^{T}\left(\boldsymbol{S}_{j}(k+1)\right)^{-1}
\end{gathered}
$$

The covariance estimation for every model can be represented as

$$
\boldsymbol{P}_{j}^{\text {pre }}(k+1)=\left(\boldsymbol{I}-\boldsymbol{K}_{j}(k+1) \boldsymbol{H}_{j}\right) \boldsymbol{P}_{j}^{\prime}(k+1)
$$

At last, the predicted covariance matrix is given as

$$
\boldsymbol{P}^{\text {pre }}(k+1)=\sum_{j=1}^{r} \boldsymbol{\mu}_{j}(k)\left(\boldsymbol{P}_{j}^{\text {pre }}(k)\right)
$$

where $\mu_{j}(k)$ is model probability at time $k$. 
4.3 Dwelling time allocation algorithm based on PSO

The desired tracking covariance matrix $\boldsymbol{P}_{\text {des }}$ should be set for the $m$ radars in the network first. Then, the dwelling time of every radar is selected as the particles in the PSO, and then the minimum and maximum ranges, velocities are designed according to the radars' performance. Initial locations and velocities of all particles are generated randomly in whole search space.

Then, the fitness function is designed as

$$
f=\min \left(\operatorname{trace}\left(\boldsymbol{P}^{\text {pre }}(k+1)-\operatorname{trace}\left(\boldsymbol{P}_{d e s}\right)\right)\right)
$$

During the PSO optimization, every radar in the network will select the dwelling time $T_{d w}{ }^{m}$, which leads to the minimum fitness function. Then, a dwelling time set can be given as

$$
\boldsymbol{T}_{d w}=\left\{\boldsymbol{T}_{d w}{ }^{1}, \boldsymbol{T}_{d w}{ }^{2} \ldots, \boldsymbol{T}_{d w}{ }^{m}\right\}
$$

To save most radiation time and get the best LPI ability of the radar network, the algorithm will choose the radar which will radiate the minimum dwelling time in the network for tracking at time $k$.

\section{SIMULATION RESULTS}

In this section, Monte Carlo simulations are performed to analyze the performance of the proposed dwelling time scheduling algorithm based on the PSO and predicted covariance (PSO-PC). The IMM filter here comprises constant velocity (CV) model and coordinated turn (CT) rate model [7].

\subsection{Trajectory design}

Figure 1 shows the target trajectory in 100s. There are three radars, which are labeled A, B and C in the radar network. The positions of the radars are $(0.80),(250.10)$ and $(-70,400)$, respectively.

\subsection{Comparison of tracking performance}

The proposed dwelling time design method (PSO-PC) of three radars is realized in the simulation, which is also compared with the performance of every single radar using the scheduling strategy of dwelling time in [6]. The root mean square error (RMSE) of time $k$ and the average rootmean-square error (ARMSE) of the whole tracking process can be formulated as (34) and (35), respectively:

$$
\begin{gathered}
\boldsymbol{R M S E}(k)=\sqrt{\frac{1}{M_{c}} \sum_{m=1}^{M_{c}}\left(x_{k}-\hat{x}_{k}^{m}\right)^{2}} \\
A R M S E=\frac{1}{N_{t}} \sum_{k=1}^{N_{t}} \boldsymbol{R M S E}(k)
\end{gathered}
$$

where $M_{c}$ is the number of the Monte Carlo simulation, $x_{k}$ is the true state of the system, $\hat{x}_{k}^{m}$ is the estimated vector at the $m$ th simulation, and $N_{t}$ is the total tracking time in every simulation. In the simulation, $M_{c}=100$ and $N_{t}=100$.

Figure 2 shows the range RMSE of the proposed method during the tracking. Figure 3(a) and (b) shows the RMSE of the proposed method in $X$ and $Y$ direction, respectively. Table 1 shows the ARMSE of the range, $X$ direction, and $Y$ direction. Compared with every radar, we can see that the proposed method of PSO-PC presents almost the same excellent tracking accuracy. 


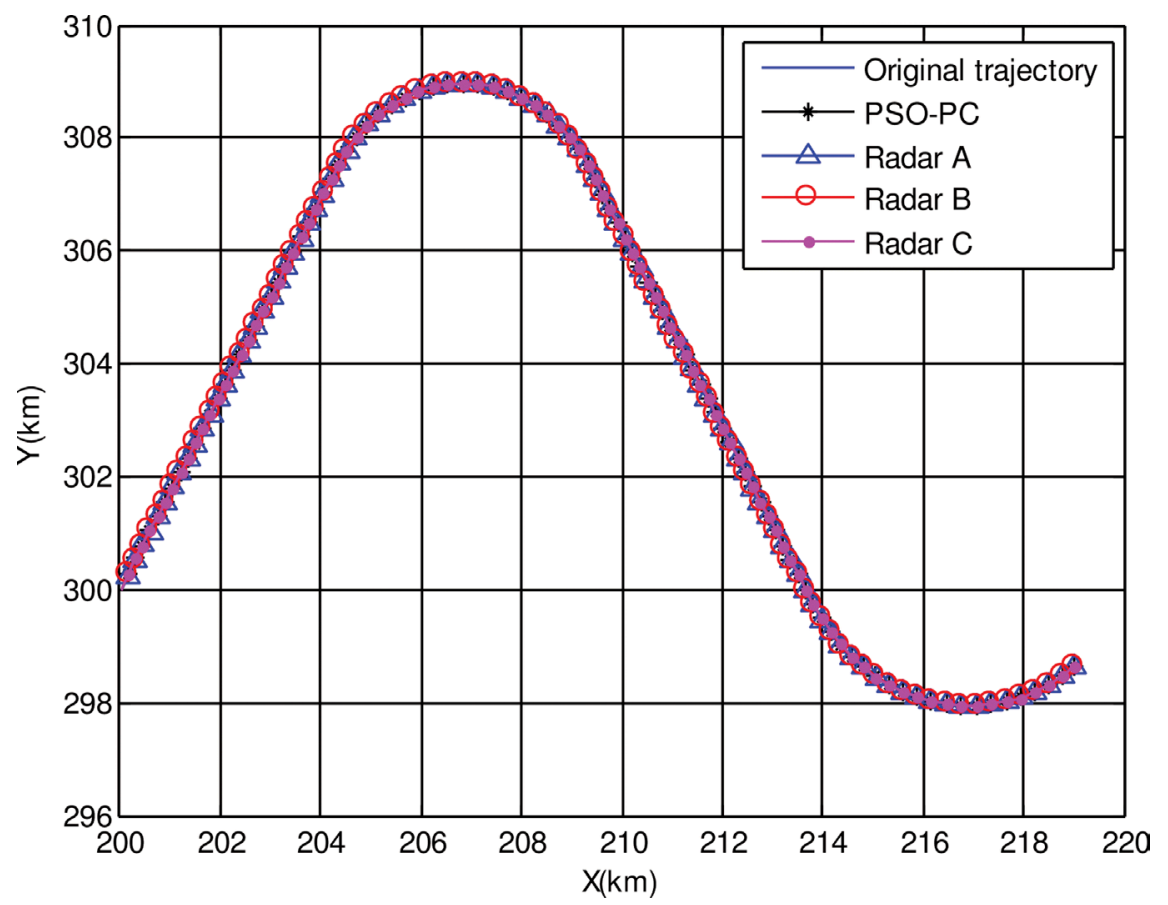

Figure 1: Trajectory of the target.

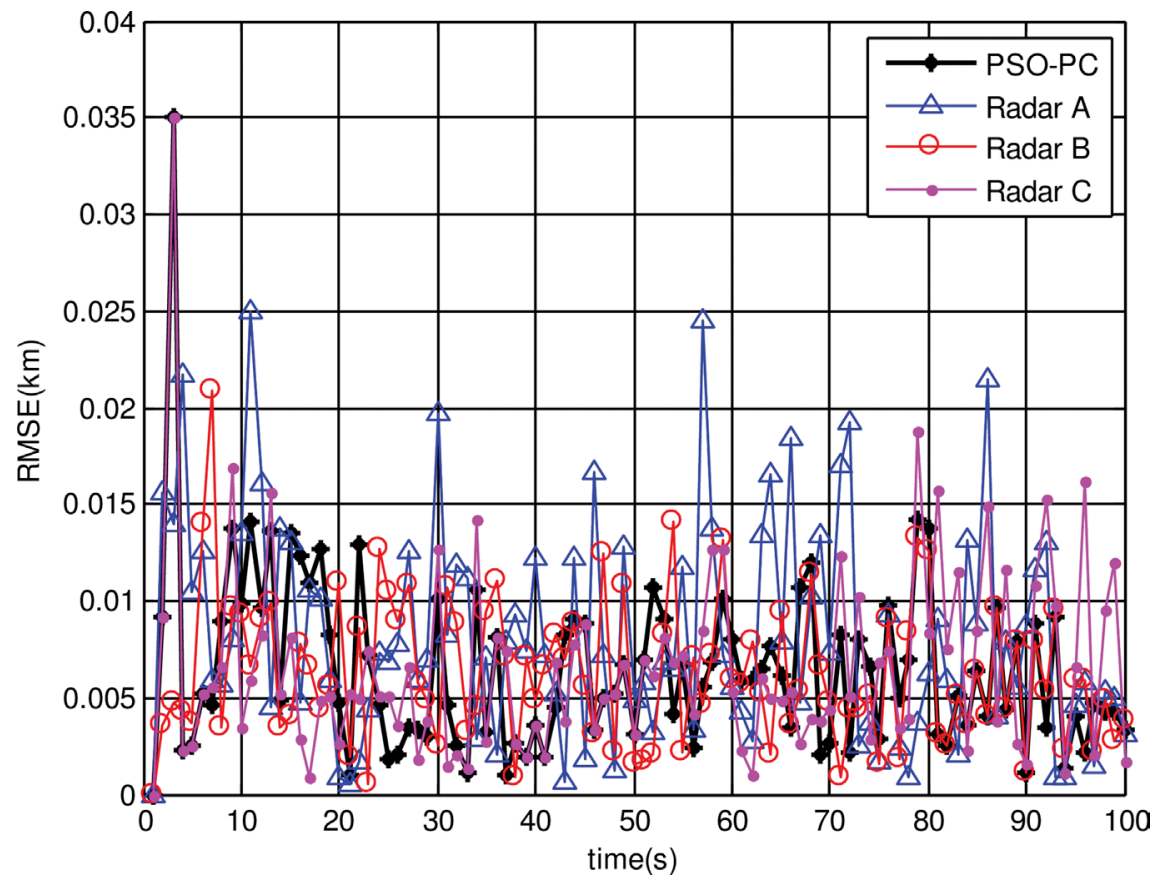

Figure 2: Comparison of tracking performance. 
(a)

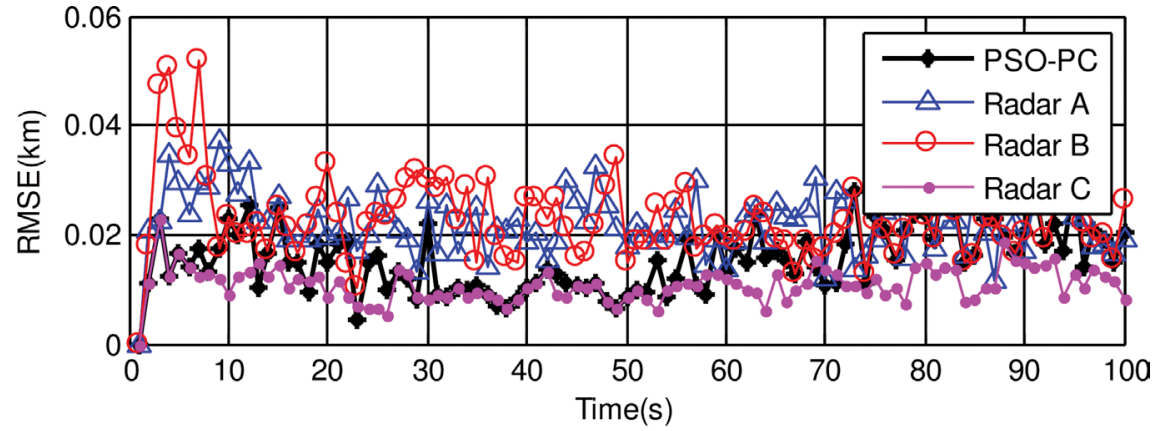

(b)

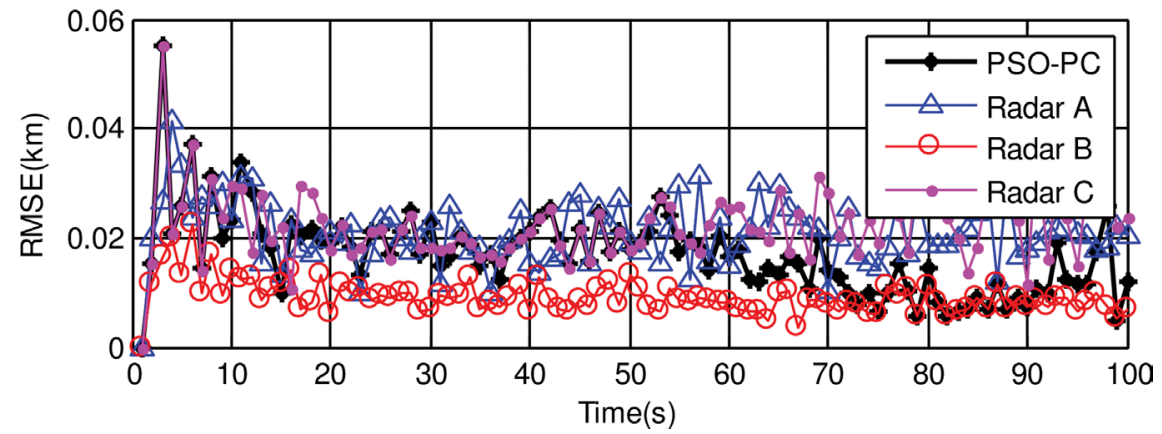

Figure 3: Comparison of tracking performance: (a) tracking performance of $\mathrm{X}$ direction and (b) tracking performance of Y direction.

Table 1: Comparison of ARMSE.

\begin{tabular}{lccc}
\hline Method & R-ARMSE $(\mathrm{km})$ & X-ARMSE $(\mathrm{km})$ & Y-ARMSE $(\mathrm{km})$ \\
\hline PSO-PC & $\mathbf{0 . 0 0 6 5}$ & $\mathbf{0 . 0 1 5 5}$ & $\mathbf{0 . 0 1 6 9}$ \\
Radar A & 0.0083 & 0.0224 & 0.0210 \\
Radar B & 0.0063 & 0.0229 & 0.0091 \\
Radar C & 0.0066 & 0.0109 & 0.0223 \\
\hline
\end{tabular}

\subsection{Comparison of dwelling time}

As dwelling time of PSO-PC, Radar A, Radar B and Radar C is shown in Fig. 4, we can see that the proposed method not only presents excellent tracking accuracy but also reduces more radiated time. As Fig. 5 shows the radiation label of the radars, we can see the radars work in turn in order to obtain excellent tracking and LPI performance.

\section{CONCLUSIONS}

In this paper, we have presented a new strategy of dwelling time allocation in the radar network based on the PSO method and predicted covariance theory. According to the optimization results of every radar, the radar with the minimum dwelling time in the network will be selected to track the 


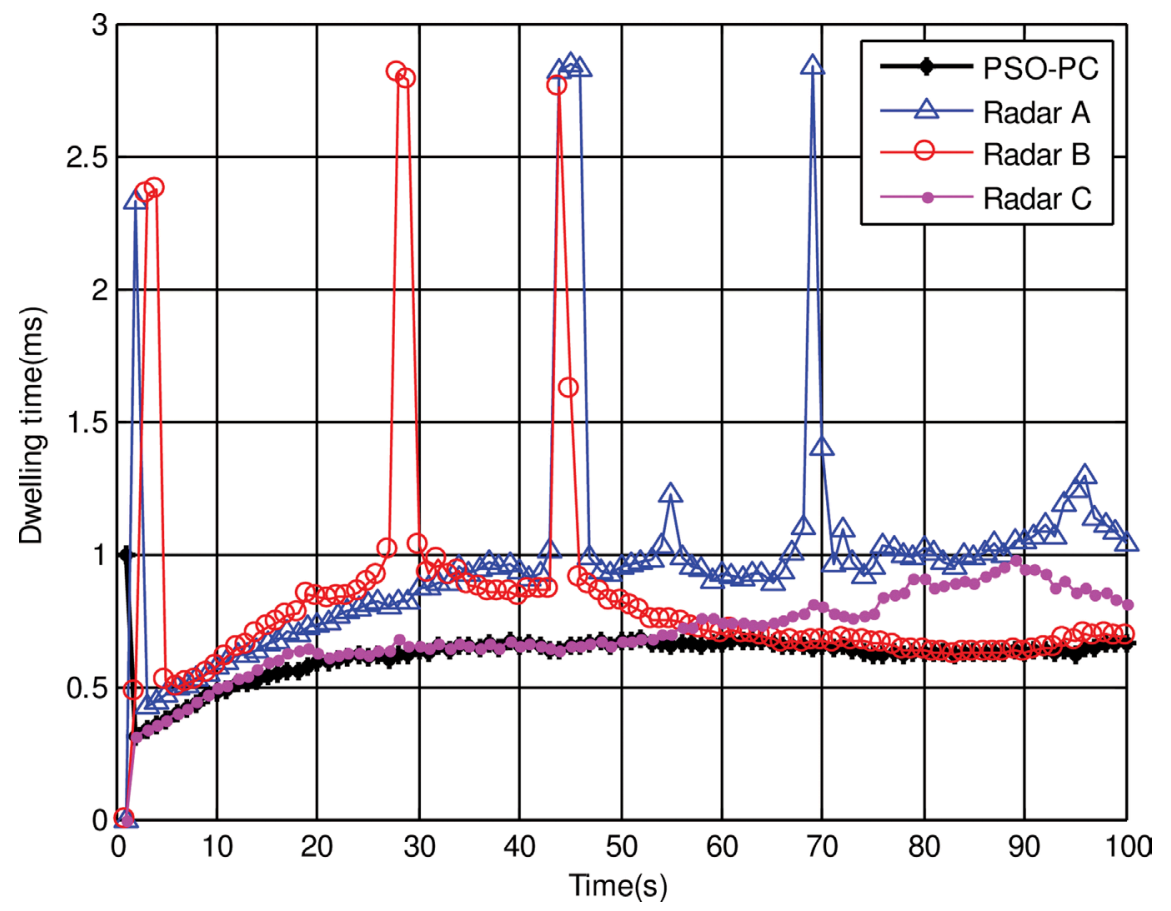

Figure 4: Comparison of dwelling time.

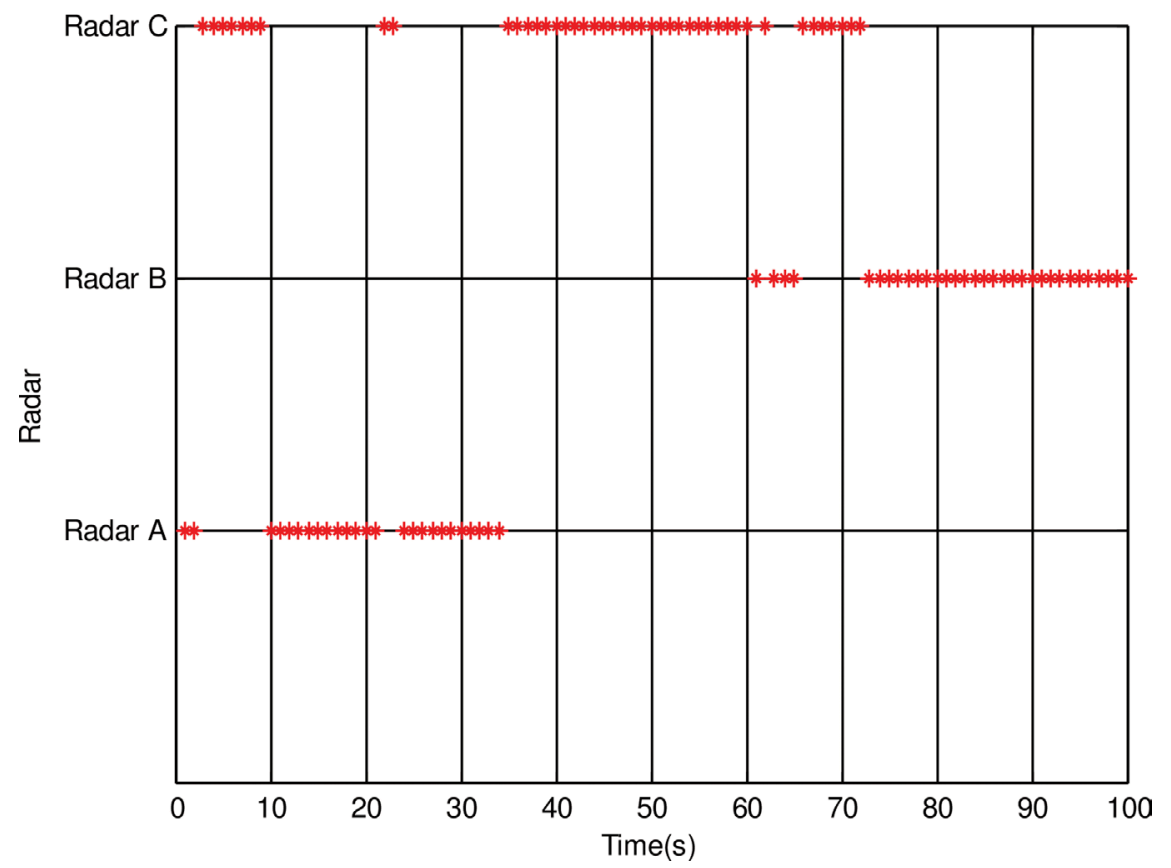

Figure 5: Radiation label of the radars. 
target. The simulation results show that the proposed method can save much more dwelling time with excellent tracking accuracy for tracking single target in the network. As for the future work, this algorithm can be modified to track more targets in complex radar network.

\section{ACKNOWLEDGEMENTS}

This work was supported by the National Natural Science Funds Fund (61401179), Colleges of Jiangsu Province Natural Science Fund (14KJB510009), the Science and Technology on Electronic Information Control Laboratory Project, Scientific Research Start-up Funding from Jiangsu University of Science and Technology, Fundamental Research Funds for the Central Universities (NJ20140010), and a Project Funded by the Priority Academic Program Development of Jiangsu Higher Education Institutions.

\section{REFERENCES}

[1] Krishnamurthy, V., Emission management for low probability intercept sensors in network centric warfare. IEEE Transactions on Aerospace and Electronics Systems, 41(1), pp. 133-151, 2005. http://dx.doi.org/10.1109/taes.2005.1413752

[2] Kuo, T.-W., Chao, Y.-S., Kuo, C.-F. \& Chang, C., Real-time dwell scheduling of componentoriented phased array radars. IEEE Transactions on Computers, 54(1), pp. 47-60, 2005. http://dx.doi.org/10.1109/tc.2005.10

[3] Mir, H.S. \& Guitouni, A., Variable dwell time task scheduling for multifunction radar. IEEE Transactions on Automation Science and Engineering, 11(2), pp. 463-472, 2014. http://dx.doi.org/10.1109/tase.2013.2285014

[4] Glass, J.D., Blair, W.D. \& Bar-Shalom, Y., Optimizing radar signal to noise ratio for tracking manoeuvring targets. 2014 17th International Conference on Information Fusion (FUSION), Salamanca, 7-10 July, 2014, pp. 1-7.

[5] Jang, D.-S., Choi, H.-L. \& Roh, J.-E., A time-window-based task scheduling approach for multifunction phased array radars. 2011 11th International Conference on Control, Automation and Systems (ICCAS), Gyeonggi-do, 26-29 October, 2011, pp. 1250-1255.

[6] Severson, T.A. \& Paley, D.A., Distributed multitarget search and track assignment with consensus-based coordination. IEEE Sensors Journal, 15(2), pp. 864-875, 2015. http://dx.doi.org/10.1109/jsen.2014.2355200

[7] Zhang, Z., Zhou, J., Wang, F., Liu, W. \& Yang, H., Multiple-target tracking with adaptive sampling intervals for phased-array radar. Journal of Systems Engineering and Electronics, 22(5), pp. 760-766, 2011.

[8] Kennedy, J. \& Eberhart, R., Particle swarm optimization. Proceedings of IEEE International Conference on Neural Networks, April, 1995, pp. 1942-1948. 